Neurosurg Focus 11 (2):Introduction, 2001 Click here to return to Table of Contents

\title{
HISTORY OF NEUROLOGICAL SURGERY
}

\author{
Neurosurgical Focus, August 2001 \\ Volume 11, Issue 2
}

Topic Editor: Michael Schulder, M.D.

The Neurological Institute of New Jersey, Newark, New Jersey

Definition of a disease, the evolution of its treatment, the mavericks who have advanced the field - examination of these phenomena allows us to look at the history of neurological surgery. This edition of Neurosurgical Focus examines key developments in our field from these perspectives.

The placement of shunts in the treatment of hydrocephalus has become one of the most common (and least beloved) procedures in neurosurgery. But for millennia increased intracranial pressure (ICP) was first an undefined, then poorly understood, and then untreatable condition. The fits, starts, and dead ends of surgery for hydrocephalus are described in our first article. Neurosurgeons also treat another form of increased ICP, most commonly known as pseudotumor cerebri (PTC). Patients with this condition often present difficult challenges; the efforts to define PTC, undertaken not all that long ago, are worth reflecting on when faced with a possibly malfunctioning lumboperitoneal shunt.

We are seeing great changes in the management of patients with cerebral aneurysms, especially with ongoing advances in endovascular techniques. Most likely, however, neurosurgeons will continue to perform craniotomies for the exclusion of aneurysms from the normal cerebral circulation. The articles in which the authors describe how we came to our current consensus on the timing of aneurysm surgery, as well as the continuing changes in aneurysm clip technology, are reminders of how much has changed in so short a time.

The contributions of two individuals are reported in this issue. Ibn Sina (known to some as Avicenna) was a Persian doctor and one of the great intellects of medieval Islamic culture. His study of anatomy included descriptions of the brain and spinal cord structure and function and is worth remembering as one of the foundations of modern medicine and neuroscience. It is interesting to speculate, as the authors do, as to how he may have performed trephinations.

Lastly, the work of Irving Cooper, a colorful and controversial pioneer in movement disorder surgery, is presented by Couldwell and Rovit who present outstanding pre-, intra-, and postoperative video footage of many of Cooper's patients. A TV interview with Cooper is included as well. Anyone with even the least experience and interest in stereotaxy must read this article and view the footage.

The history of neurosurgery is as old as antiquity and as new as yesterday. I hope these articles will interest and help readers to reflect on our past and consider our future.

Michael Schulder, M.D. 